\title{
Immersion Anomaly of Dirac Operator on Surface in $\mathbb{R}^{3}$
}

\author{
Shigeki MATSUTANI \\ 2-4-11 Sairenji, Niihama, Ehime, 792 Japan
}

\begin{abstract}
In previous report (J. Phys. A (1997) 30 4019-4029), I showed that the Dirac field confined in a surface immersed in $\mathbb{R}^{3}$ by means of a mass type potential is governed by the KonopelchenkoKenmotsu-Weierstrass-Enneper equation. In this article, I quantized the Dirac field and calculated the gauge transformation which exhibits the gauge freedom of the parameterization of the surface. Then using the Ward-Takahashi identity, I showed that the expectation value of the action of the Dirac field is expressed by the Willmore functional and area of the surface.
\end{abstract}

\section{$\S 1$. Introduction}

In the previous report [1], I showed that the Dirac field confined in a thin curved surface $S$ immersed in three dimensional flat space $\mathbb{R}^{3}$ obeys the Dirac equation which is discovered by Konopelchenko [2-4]

$$
\partial f_{1}=p f_{2}, \quad \bar{\partial} f_{2}=-p f_{1},
$$

where

$$
p:=\frac{1}{2} \sqrt{\rho} H,
$$

$H$ is the mean curvature of the surface $S$ parameterized by complex $z$ and $\rho$ is the factor of the conformal metric induced from $\mathbb{R}^{3}$.

This equation completely represents the immersed geometry as the old Weierstrass-Enneper equation expresses the minimal surface [2].

Even though the relation had been essentially found by Kenmotsu [4-7], the formulation as the Dirac type was performed by Konopelchenko and recently it is revealed that the Dirac operator has more physical and mathematical meanings; the Dirac operator is a translator between the geometrical and analytical objects [8] even in the arithmetic geometry of the number theory [9]. Thus although the Dirac type equation (1-1) has been called as the generalized Weierstrass equation, in this article I will call it Konopelchenko-Kenmotsu-Weierstrass-Enneper (KKWE) equation.

The immersion geometry is currently studied in the various fields, e.g., soliton theory, the differential geometry, the harmonic map theory, string theory and so on. In the soliton theory, the question what is the integrable is the most important theme and one of its answers might be found in the immersed geometry. In fact, the static sine-Gordon equation was discovered by Euler, from the energy functional of the elastica given by Daniel Bernoulli in eighteenth century, as an elastica immersed in $\mathbb{R}^{2}[10]$ and the net sine-Gordon equation was found in the last century as a surface immersed in $\mathbb{R}^{3}$ [11]. Recently Goldstein and Petrich discovered the modified KdV (MKdV) hierarchy by considering one parameter deformation of a space curve immersed in $\mathbb{R}^{2}[12,13]$. After their new interpretation, there appear several geometrical realizations of the soliton theory [14-16]. In the differential geometry, after the discovery the exotic solution of the constant mean curvature surface by Wente [17], the extrinsic structure is currently studied again [18-20]. In the harmonic map theory, 
extrinsic topology [20] like the elastica problem [21], a prototype of such model which firstly entered in history [10].

Furthermore Polyakov introduced an extrinsic action in the string theory and the theory of 2dimensional gravity for renomalizability [22]. His program has been studied in the framework of W-algebra [23] but recently was investigated by Carroll and Konopelchenko [24] and Viswanathan and Parthasarathy [25] using more direct method. Polyakov's extrinsic action in the classical level is the same as the Willmore functional [26,27],

$$
W=\int_{\mathcal{S}} \mathrm{dvol} H^{2}
$$

where "dvol" is a volume form of the surface $S$.

Accordingly the immersed surface is a very current object and its studies currently progress.

On the other hand I have been studying the Dirac field confined in an immersed object and its relationship with the immersed object itself [28-32]. Since the Dirac operator should be regarded as a translator (a functor) between the analytical object and the geometrical object [8-9], in terms of the Dirac operator, I have been studying the physical and geometrical meanings of the abstract theorems in the soliton theory and quantum theory focusing on the elastica problem [28-32] and recently on the immersed surface [1].

In this article, I will deal with the quantized Dirac field and investigate the gauge freedom which does not change the Willmore functional. In other words, I will search for a symmetry in the classical level and compute its anomalous relation in quantum level using Fujikawa type prescription [33-35]. Furthermore since there are negative eigenvalues which makes the theory worse in a calculus, I will propose new regularization which can be regarded as a local version of a generalization of Hurwitz $\zeta$-function [36] and then I will obtain the finite result and the coupling constant of the Liouville action $[22]$ as convergent parameter. Finally I have the relation between the expectation value of the action of the Dirac field and the Willmore functional. It reminds me of the boson-fermion correspondence in this system. It should be noted that even though it does not directly generate topological index, it could be regarded as a local version of the Atiyah-Singer type index theorem [8,9,37-39].

Organization of this article is as follows. Section 2 reviews the extrinsic geometry of a surface immersed in $\mathbb{R}^{3}$. There I will introduce the Willmore surface as a free energy of a thin elastic surface. In section 3, starting with the quantized Dirac field whose on-shell equation is KKWE equation (1-1), I will calculate the variation of a gauge transformation. Using the Ward-Takahashi identity, I will obtain an anomalous relation exhibiting this system. In section 4, I will discuss the obtained results.

\section{$\S 2$. Conformal Surface Immersed in $\mathbb{R}^{3}$}

In this article, I will consider a compact surface $\mathcal{S}$ immersed in $\mathbb{R}^{3}$, which has a complex structure [1-4],

$$
\varpi: \Sigma \rightarrow \mathcal{S} \subset \mathbb{R}^{3},
$$

where $\Sigma$ and $\mathcal{S}$ are two-dimensional conformal manifold. $\mathcal{S}$ is parameterized by two-dimensional coordinate system $\left(q^{1}, q^{2}\right) \in \Sigma$.

A position on a conformal compact surface $\mathcal{S}$ is represented using the affine vector $\mathbf{x}\left(q^{1}, q^{2}\right)=\left(x^{I}\right)$ $=\left(x^{1}, x^{2}, x^{3}\right)$ in $\mathbb{R}^{3}$ and the normal unit vector of $\mathcal{S}$ is denoted by $\mathbf{e}_{3}$. I sometimes regard the euclidean space as a product manifold of complex plane and real line, $\mathbb{R}^{3} \approx \mathbb{C} \times \mathbb{R}[1-4]$,

$$
Z:=x^{1}+\mathrm{i} x^{2} \in \mathbb{C}, \quad x^{3} \in \mathbb{R} .
$$

The surface $\mathcal{S}$ has the conformal flat metric, 
The complex parameterization of the surface is employed,

$$
z:=q^{1}+\mathrm{i} q^{2}
$$

and

$$
\partial:=\frac{1}{2}\left(\partial_{q^{1}}-\mathrm{i} \partial_{q^{2}}\right), \quad \bar{\partial}:=\frac{1}{2}\left(\partial_{q^{1}}+\mathrm{i} \partial_{q^{2}}\right), \quad \mathrm{d}^{2} q:=\mathrm{d} q^{1} \mathrm{~d} q^{2}=: \frac{1}{2} \mathrm{~d}^{2} z:=\frac{1}{2} \mathrm{id} z \mathrm{~d} \bar{z} .
$$

For a given function $f$ over $\mathcal{S}$, if $f$ is real analytic, I denote it as $f=f(q)$ but if it should be regarded as a complex analytic function, I will use the notation, $f=f(z)$.

Then the moving frame can be written as

$$
e_{\alpha}^{I}:=\partial_{\alpha} x^{I}, \quad e_{z}^{I}:=\partial x^{I}
$$

where $\partial_{\alpha}:=\partial_{q^{\alpha}}:=\partial / \partial q^{\alpha}$. Their inverse matrices are denoted as $e_{I}^{\alpha}$ and $e_{I}^{z}$. The metric is expressed as

$$
\frac{1}{4} \rho=<\mathbf{e}_{z}, \mathbf{e}_{\bar{z}}>:=\delta_{a, b} e_{z}^{I} e^{b}{ }_{\bar{z}}
$$

Here $<,>$ denotes the canonical inner product in the euclidean space $\mathbb{R}^{3}$.

The second fundamental form is denoted as,

$$
\gamma_{\beta \alpha}^{3}:=<\mathbf{e}_{3}, \partial_{\alpha} \mathbf{e}_{\beta}>. \operatorname{tag} 2-8
$$

Using the relation $<\mathbf{e}_{3}, \partial_{\alpha} \mathbf{e}_{3}>=0$, the Weingarten map, $-\gamma_{\beta 3}^{\alpha} \mathbf{e}_{\alpha}$, is defined by

$$
\gamma_{\beta 3}^{\alpha}=<\mathbf{e}^{\alpha}, \partial_{\beta} \mathbf{e}_{3}>
$$

From $\partial_{\alpha}<\mathbf{e}^{\gamma}, \mathbf{e}_{3}>=0, \gamma_{\beta 3}^{\alpha}$ is related to the second fundamental form through the relation,

$$
\gamma_{\beta \alpha}^{3}=-\gamma_{3 \alpha}^{\gamma} g_{\gamma \beta}=-\gamma_{3 \alpha}^{\beta} \rho,
$$

is the surface metric. It is worth while noting that for a scaling of $\left(q^{1}, q^{2}\right) \rightarrow \lambda\left(q^{1}, q^{2}\right)$, the Weingarten map does not change.

In terms of the Weingarten map, I will introduce invariant quantities for the coordinate transformation if I fix the surface $\mathcal{S}$. They are known as the mean and the Gaussian curvatures on $\mathcal{S}:$

$$
H:=-\frac{1}{2} \operatorname{tr}_{2}\left(\gamma_{3 \beta}^{\alpha}\right), \quad K:=\operatorname{det}_{2}\left(\gamma_{3 \beta}^{\alpha}\right) .
$$

Here $\operatorname{tr}_{2}$ and $\operatorname{det}_{2}$ are the two-dimensional trace and determinant over $\alpha$ and $\beta$ respectively. Due to the Gauss's egregium theorem, I have the relation,

$$
K=-2 \frac{1}{\rho} \partial \bar{\partial} \log \rho,
$$

and from the properties of complex manifold, I obtain

$$
H=\frac{2}{\rho}<\partial \bar{\partial} \mathbf{x}, \mathbf{e}^{3}>=\frac{4}{\mathrm{i} \rho^{2}} \epsilon_{I J K} \partial \bar{\partial} x^{I} \partial x^{J} \bar{\partial} x^{K} .
$$

Using the independence of the choice of the local coordinate, I will introduce a proper coordinate transformation which diagonalizes the Weingarten map, 
These diagonal elements $\left(k_{1}, k_{2}\right)$ are known as the principal curvatures of the surface $\mathcal{S}$. In terms of these values, the Gauss and mean curvatures are expressed as [27],

$$
K=k_{1} k_{2}, \quad H=\frac{1}{2}\left(k_{1}+k_{2}\right) .
$$

Here I will regarded the surface $\mathcal{S}$ as a shape of a thin elastic surface. Its local free energy density is given as an invariant for the local coordinate transformation. On the other hand, the difference of the local surface densities between inside and outside surfaces is proportional to the extrinsic curvature due to its thickness, for a local deformation of the surface. By the linear response of the elastic body theory and independence of the coordinate transformation, the free energy might be given as [40]

$$
f=B_{0} H^{2}+B_{1} K=\frac{1}{4} B_{0}\left(k_{1}^{2}+k_{2}^{2}\right)+\left(B_{0}+B_{1}\right) k_{1} k_{2},
$$

where $B$ 's are elastic constants. However using the Gauss-Bonnet theorem [27], the integral of the second term in (2-16) is expressed as,

$$
\int \rho \mathrm{d}^{2} q K=\int \rho \mathrm{d}^{2} q k_{1} k_{2}=2 \pi \chi
$$

where $\chi$ is the Euler characteristic, which is an integer and exhibits the global topological properties of the surface. Hence the second term in (2-16) is not dynamical one if one fixes the topology of the system.

Hence the free energy of the system becomes

$$
W=B_{0} \int \rho \mathrm{d}^{2} q H^{2}
$$

This functional integral is known as the Willmore functional $[26,27]$ and, recently, as the Polyakov's extrinsic action in the 2-dimensional gravity [22,24,25]. For later convenience, I will fix $B_{0}=1$ and introduce a quantity [1-4],

$$
p:=\frac{1}{2} \sqrt{\rho} H=\frac{1}{2} g^{1 / 4} H
$$

Using this new quantities, the Willmore functional is written as,

$$
W=4 \int \mathrm{d}^{2} q p^{2}
$$

\section{$\S 3$. Immersion Anomaly}

In the previous report [1], I showed that the Dirac field confined in the surface $\mathcal{S}$ obeys the KKWE equation (1-1). In this article, I will deal with the quantized fermion over the immersed surfaces. As I did in ref.[29], after I quantize the Dirac field, I can obtain the quantized Dirac field over the immersed thin surface $\mathcal{S}$ by confining it using the confinement mass-type potential. This computation can be performed parallelled to the arguments in refs. [1] and [29]. Thus in this article, I will start with the quantized Dirac field of the surface $\mathcal{S}[1,22,41]$. The partition function of the Dirac field is given as,

$$
Z[\overline{\boldsymbol{\psi}}, \boldsymbol{\psi}, \rho, H]=\int D \overline{\boldsymbol{\psi}} D \boldsymbol{\psi} \exp \left(-S_{\operatorname{Dirac}}[\overline{\boldsymbol{\psi}}, \boldsymbol{\psi}, \rho, H]\right)
$$

where $[1,15,42]$ 


$$
\begin{gathered}
\not \mathcal{D}:=\gamma^{\alpha} \mathcal{D}_{\alpha}+\gamma^{3} H, \quad \mathcal{D}_{\alpha}:=\partial_{\alpha}+\omega_{\alpha}, \quad \omega_{\alpha}:=-\frac{1}{4} \rho^{-1} \sigma^{a b}\left(\partial_{a} \rho \delta_{\alpha b}-\partial_{b} \rho \delta_{\alpha a}\right), \\
\gamma^{\alpha}=e_{a}^{\alpha} \sigma^{a}, \quad \gamma^{3}=\sigma^{3}, \quad \sigma^{a b}:=\left[\sigma^{a}, \sigma^{b}\right] / 2, \\
\overline{\boldsymbol{\psi}}=\boldsymbol{\psi}^{\dagger} \sigma^{1} \rho^{1 / 2} .
\end{gathered}
$$

Here I denoted the Pauli matrix as $\sigma^{a}$ and used the conformal gauge freedom, $e_{\alpha}^{a}=\rho^{1 / 2} \delta_{\alpha}^{a}$. The indices $a, b$ is of the inner space and run over 1 and 2. The Dirac operator can be expressed as

$$
\begin{aligned}
\not \mathcal{D} & :=\left(\gamma^{\alpha} \mathcal{D}_{\alpha}+\gamma^{3} H\right) \\
& =\sigma^{a} \rho^{-1 / 2} \delta_{a}^{\alpha}\left[\partial_{\alpha}+\frac{1}{2} \rho^{-1}\left(\partial_{\alpha} \rho\right)\right]+\sigma^{3} H \\
& =2\left(\begin{array}{cc}
H / 2 & \rho^{-1 / 2} \bar{\partial} \rho^{1 / 2} \\
\rho^{-1 / 2} \partial \rho^{1 / 2} & -H / 2
\end{array}\right) .
\end{aligned}
$$

Noting the fact that $\boldsymbol{\psi}$ 's are just integral variables in the path integral, the kinetic term of the Dirac operator is hermite,

$$
\begin{aligned}
<\boldsymbol{\psi} \mid \mathrm{i}\left(\begin{array}{cc}
0 & \rho^{-1} \bar{\partial} \rho^{1 / 2} \\
\rho^{-1} \partial \rho^{1 / 2} & 0
\end{array}\right) \boldsymbol{\psi}> & =\mathrm{i} \int \rho \mathrm{d}^{2} z \overline{\boldsymbol{\psi}}\left(\begin{array}{cc}
0 & \rho^{-1} \bar{\partial} \rho^{1 / 2} \\
\rho^{-1} \partial \rho^{1 / 2} & 0
\end{array}\right) \boldsymbol{\psi} \\
& =-\mathrm{i} \int \mathrm{d}^{2} z\left(\overline{\boldsymbol{\psi}_{1}} \overleftarrow{\bar{\partial}} \rho^{1 / 2} \boldsymbol{\psi}_{2}+\overline{\boldsymbol{\psi}}_{2}^{*} \overleftarrow{\partial} \rho^{1 / 2} \boldsymbol{\psi}_{1}\right) \\
& =-\mathrm{i} \int \mathrm{d}^{2} z\left(\boldsymbol{\psi}_{2}^{*} \rho^{1 / 2} \overleftarrow{\bar{\partial}} \rho^{1 / 2} \boldsymbol{\psi}_{2}+\boldsymbol{\psi}_{1} \rho^{1 / 2} \overleftarrow{\partial} \rho^{1 / 2} \boldsymbol{\psi}_{1}\right) \\
& =\int \rho \mathrm{d}^{2} z\left(\mathrm{i}\left(\begin{array}{cc}
0 & \rho^{-1} \partial \rho^{1 / 2} \\
\rho^{-1} \bar{\partial} \rho^{1 / 2} & 0
\end{array}\right)\right)^{\dagger} \sigma^{1} \rho^{1 / 2} \boldsymbol{\psi} \\
& \sim<\mathrm{i}\left(\begin{array}{cc}
\rho^{-1} \bar{\partial} \rho^{1 / 2} \\
\rho^{-1} \partial \rho^{1 / 2} & 0
\end{array}\right) \boldsymbol{\psi} \mid \boldsymbol{\psi}>
\end{aligned}
$$

As I showed in ref.[1], when I redefine the Dirac field in the surface $\mathcal{S}$ as

$$
f:=\rho^{1 / 2} \boldsymbol{\psi}
$$

the Dirac operator becomes simpler,

$$
\begin{aligned}
\mathcal{L}_{f} \sqrt{g} \mathrm{~d}^{2} q & =\mathrm{i} f^{\dagger} \sigma^{1}\left(\sigma^{a} \delta_{a}^{\alpha} \partial_{\alpha}+\rho^{1 / 2} H \sigma^{3}\right) f \mathrm{~d}^{2} q \\
& =\mathrm{i} \bar{f}\left(\begin{array}{cc}
p & \bar{\partial} \\
\partial & -p
\end{array}\right) f \mathrm{~d}^{2} z
\end{aligned}
$$

where $p$ is defined in $(2-21)$

Then the KKWE equation is obtained as the on-shell motion of (3-9) [1-4],

$$
\partial f_{1}=p f_{2}, \quad \bar{\partial} f_{2}=-p f_{1} .
$$

These equations which were found by Konopelchenko reproduce all properties of the extrinsic geometry of this system. Their properties were studied by Konopelchenko and Taimanov [2-4,6,7],

$$
f_{1}=\sqrt{\mathrm{i} \bar{\partial} \bar{Z} / 2}, \quad f_{2}=\sqrt{-\mathrm{i} \partial \bar{Z} / 2}
$$

This relation may be interpreted as the bosonaization in the conformal field theory [22]. It should be noted that its lower dimensional version of the KKWE equation is found through study of the 
The Willmore functional (2-20) is expressed by $p$ and $p$ consists of multiple of $H$ and $\sqrt{\rho}$. Hence by fixing $p$, there still remains a freedom of choice of $\rho$; fixing $p$ means the deformation of $\rho$ without changing the Willmore functional. Corresponding to the deformation preserving the value of the Willmore functional, the lagrangian of the Dirac field (3-2) has a similar gauge freedom which does not change the action $S_{\text {Dirac }}$. In fact using such the gauge freedom, I scaled the Dirac field $\boldsymbol{\psi}$ to $f$ in (3-8).

However in the quantum field theory, even though the lagrangian is invariant for a transformation, the partition function is not in general due to the jacobian of the functional measure. The purpose of this article is to calculate this quantum effect. Thus I will estimate the infinitesimal gauge transformation which does not change the action of the Dirac field (3-2) and is an analogue of the transformation of (3-8).

Following a conventional notation, I will introduce the dilatation parameter,

$$
\phi:=\frac{1}{2} \log \rho,
$$

which is sometimes called as dilaton [22]. Furthermore I will rewrite the Dirac operator in (3-6) as,

$$
\not D=2\left(\begin{array}{cc}
p \rho^{-1 / 2} & \rho^{-1} \bar{\partial} \rho^{1 / 2} \\
\rho^{-1} \partial \rho^{1 / 2} & -p \rho^{-1 / 2}
\end{array}\right) .
$$

As I mentioned above, I will deal with the variation of the dilaton preserving $p$,

$$
\phi \rightarrow \phi+\alpha, \quad\left(\rho \rightarrow \rho \mathrm{e}^{2 \alpha}\right), \quad p \rightarrow p .
$$

For the infinitesimal variation of the dilaton, the action of the fermionic field changes its value,

$$
\mathcal{S}_{\text {Dirac }} \rightarrow \mathcal{S}_{\text {Dirac }}{ }^{\prime}=\mathcal{S}_{\text {Dirac }}+\mathrm{i} \int \rho \mathrm{d}^{2} q \alpha\left(\rho^{-1} \delta_{a}^{\beta} \partial_{\beta} \overline{\boldsymbol{\psi}} \sigma^{a} \rho^{1 / 2} \boldsymbol{\psi}+\overline{\boldsymbol{\psi}} \not \boldsymbol{\psi}\right)
$$

However this change can be classically canceled out by the gauge transformation,

$$
\boldsymbol{\psi} \rightarrow \boldsymbol{\psi}^{\prime}=\mathrm{e}^{-\alpha} \boldsymbol{\psi}, \quad \overline{\boldsymbol{\psi}} \rightarrow \overline{\boldsymbol{\psi}}^{\prime}=\overline{\boldsymbol{\psi}}
$$

In other words, I have the identity,

$$
S_{\text {Dirac }}\left[\overline{\boldsymbol{\psi}}, \boldsymbol{\psi}, \rho^{\prime}, p\right] \rightarrow S_{\text {Dirac }}\left[\overline{\boldsymbol{\psi}}^{\prime}, \boldsymbol{\psi}^{\prime}, \rho^{\prime}, p\right]=S_{\text {Dirac }}[\overline{\boldsymbol{\psi}}, \boldsymbol{\psi}, \rho, p]
$$

Here I will evaluate the variations (3-14) and (3-16) in the framework of the quantum theory $[30,31,33]$,

$$
\begin{aligned}
Z\left[\rho^{\prime}, H^{\prime}\right] & =\int \mathrm{D} \overline{\boldsymbol{\psi}} \mathrm{D} \boldsymbol{\psi} \exp \left(-S_{\text {Dirac }}\left[\overline{\boldsymbol{\psi}}, \boldsymbol{\psi}, \rho^{\prime}, p\right]\right)=: Z_{1} \\
& =\int D \overline{\boldsymbol{\psi}}^{\prime} \mathrm{D} \boldsymbol{\psi}^{\prime} \exp \left(-S_{\text {Dirac }}\left[\overline{\boldsymbol{\psi}}^{\prime}, \boldsymbol{\psi}^{\prime}, \rho^{\prime}, p\right]\right) \\
& =\int D \overline{\boldsymbol{\psi}} \mathrm{D} \boldsymbol{\psi} \frac{\delta \boldsymbol{\psi} \delta \overline{\boldsymbol{\psi}}}{\delta \boldsymbol{\psi}^{\prime} \delta \overline{\boldsymbol{\psi}}^{\prime}} \exp \left(-S_{\text {Dirac }}[\overline{\boldsymbol{\psi}}, \boldsymbol{\psi}, \rho, p]\right)=: Z_{2}
\end{aligned}
$$

Noting that $\boldsymbol{\psi}$ 's are grassmannian variables, the jacobian is given as $(\delta \psi \delta \bar{\psi}) /\left(\delta \psi^{\prime} \delta \bar{\psi}^{\prime}\right)$. In order to compute these variations (3-18), I will introduce complete sets associated with this system $[29,30,34]$; 
and

$$
\int \rho \mathrm{d}^{2} q \chi_{m}^{\dagger}(q) \varphi_{n}(q)=\delta_{m, n}
$$

Then the variation of the field is expressed as

$$
\psi^{\prime}=: \sum_{m} a_{m}^{\prime} \varphi_{m}=\sum_{m} e^{-\alpha} a_{m} \varphi_{m}
$$

Here I will evaluate the fermionic jacobian in the transformations,

$$
\begin{aligned}
a_{m}^{\prime} & =\sum_{n} \int \rho \mathrm{d}^{2} q \chi_{m}^{\dagger} e^{-\alpha} \varphi_{n} a_{n} \\
& =: \sum_{n} \mathcal{C}_{m, n} a_{n} .
\end{aligned}
$$

The change of the functional measure is expressed by $[29,30,32,33]$,

$$
\prod_{m} \mathrm{~d} a_{m}^{\prime}=\left[\operatorname{det}\left(\mathcal{C}_{m, n}\right)\right]^{-1} \prod_{m} \mathrm{~d} a_{m}
$$

By calculation, the jacobian is written by more explicit form,

$$
\begin{aligned}
{\left[\operatorname{det}\left(\mathcal{C}_{m, n}\right)\right]^{-1} } & =\left[\operatorname{det}\left(\delta_{m, n}-\int \rho \mathrm{d}^{2} q \alpha \chi_{m}^{\dagger}(q) \varphi_{n}(q)\right)\right]^{-1} \\
& =\exp \left[\sum_{m} \int \rho \mathrm{d}^{2} q \alpha \chi_{m}^{\dagger}(q) \varphi_{m}(q)\right] \\
& =: \exp \left[\int \rho \mathrm{d}^{2} q \alpha \mathcal{A}(q)\right] .
\end{aligned}
$$

Since $\mathcal{A}(q)$ is not well-defined and unphysically diverges, I must regularize it. In this article, I will employ the modified negative power kernel regularization procedure which is partially proposed by Alves et al. [35] and is a local version of the Hurwitz $\zeta$ regularization [8,36,39]. However the Dirac operator $\not \mathcal{D}$ is not hermite and the real part of some of the eigenvalues of $-\not^{2}$ are negative. Hence I cannot directly apply negative power regularization of Alves et al. [35]. Even though the heat kernel function can be adapted for such Dirac operator with negative eigenvalues [39], $A(q)$ is not completely regularized by the heat kernel as Alves et al pointed out [35]. Thus by generalizing Hurwitz $\zeta$ function [36] rather than the Riemann $\zeta$ function, I will modify the negative power regularization [35].

I will introduce a finite positive parameter

$$
\mu^{2}>-\min _{n}\left(\operatorname{Re} \lambda_{n}^{2}\right) \geq 0
$$

and let the modified negative power kernel and the modified heat kernel defined as $[8,35,38,41]$,

$$
\mathcal{K}_{\zeta}(q, r, s \mid \mu)=\sum_{m}\left(\lambda_{m}^{2}+\mu^{2}\right)^{-s} \varphi_{m}(q) \chi_{m}^{\dagger}(r), \quad \mathcal{K}_{\mathrm{HK}}(q, r, \tau \mid \mu)=\sum_{m} \mathrm{e}^{-\left(\lambda_{m}^{2}+\mu^{2}\right) \tau} \varphi_{m}(q) \chi_{m}^{\dagger}(r)
$$

The both are connected by the Mellin transformation [35],

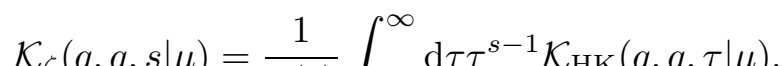


From the definition, all quantities $\lambda_{m}^{2}+\mu^{2}$ are positive, the integration in (3-27) is well-defined. If I also trace (integrate) $\mathcal{K}_{\zeta}(q, q, s \mid \mu)$ over the space-time $q$, it is just a generalized $\zeta$-function, which is generalization of Hurwitz $\zeta$ function for the Dirac operator,

$$
\zeta(s, \mu)=\sum_{m} \frac{1}{\left(\lambda_{m}^{2}+\mu^{2}\right)^{s}} .
$$

Then $\mathcal{A}(q)$ should be redefined,

$$
\mathcal{A}(q) \equiv \lim _{s \rightarrow 0} \lim _{r \rightarrow q} \operatorname{tr} \mathcal{K}_{\zeta}(q, r, s \mid \mu)
$$

For small $\tau$, the heat kernel $K_{\mathrm{HK}}$ is asymptotically expanded as [8,38],

$$
\mathcal{K}_{\mathrm{HK}}(q, r, \tau \mid \mu) \sim \frac{1}{4 \pi \tau} \mathrm{e}^{-(q-r)^{2} / 4 \tau} \sum_{n=0}^{\infty} e_{n}(q, r) \tau^{n}
$$

Accordingly I calculate $\mathcal{K}_{\zeta}(q, q, s \mid \mu)$ as [35],

$$
\begin{aligned}
K_{\zeta}(q, q, s \mid \mu) & =\frac{1}{\Gamma(s)}\left(\int_{0}^{\epsilon} \mathrm{d} \tau \tau^{s-1} \mathcal{K}_{\mathrm{HK}}(q, q, \tau \mid \mu)+\int_{\epsilon}^{\infty} \mathrm{d} \tau \tau^{s-1} \mathcal{K}_{\mathrm{HK}}(q, q, \tau \mid \mu)\right) \\
& =\frac{1}{\Gamma(s)}\left(\int_{0}^{\epsilon} \mathrm{d} \tau \tau^{s-1}\left(\frac{1}{4 \pi \tau} \sum_{n} e_{n} \tau^{n}+\int_{\epsilon}^{\infty} \mathrm{d} \tau \tau^{s-1} \mathcal{K}_{\mathrm{HK}}(q, q, \tau \mid \mu)\right)\right. \\
& =\frac{1}{\Gamma(s+1)}\left(\frac{1}{4 \pi \tau} \sum_{n} e_{n} \frac{\epsilon^{n-1}}{s-1+n}+s G(s)\right) .
\end{aligned}
$$

Here I used $\Gamma(s+1)=s \Gamma(s)$. Since $K_{\mathrm{HK}}(q, q, \tau \mid \mu) \propto \exp (-\lambda \tau)$ as $\tau \rightarrow \infty(\lambda>0)$, the second term is a certain entire analytic function over the $s$-plane and I denoted it $G(s)$. Noting $\Gamma(1)=1,(3-29)$ turns out

$$
\mathcal{A}(q)=\frac{1}{4 \pi} e_{1} .
$$

On the other hand, according to ref. [39], since the square of the Dirac operator (3-12) is given as

$$
-\not^{2}=\rho^{-1}\left(\begin{array}{cc}
-4 \bar{\partial} \partial+2 \rho^{-1}(\partial \rho) \bar{\partial}+\left(K \rho-4 p^{2}\right) & -4 \rho^{1 / 2}\left(\bar{\partial} p \rho^{-1 / 2}\right) \\
-4 \rho^{1 / 2}\left(\partial p \rho^{-1 / 2}\right) & -4 \partial \bar{\partial}+2 \rho^{-1}(\bar{\partial} \rho) \partial+\left(K \rho-4 p^{2}\right)
\end{array}\right),
$$

the coefficient of the expansion (3-30) is written by,

$$
\begin{aligned}
e_{1} & =4 p^{2} \rho^{-1}-\mu^{2}+2 \rho^{1 / 2} \sigma^{a} \delta_{a}{ }^{\beta} \partial_{\beta} p \rho^{-1 / 2}-\frac{5}{6} K \\
& =4 p^{2} \rho^{-1}-\mu^{2}-\frac{5}{6} K+2 \rho^{1 / 2} \sigma^{a} \delta_{a}^{\beta} \partial_{\beta} p \rho^{-1 / 2}
\end{aligned}
$$

Noting the fact that trace over the spin index generates the functor 2, I obtain,

$$
\mathcal{A}(q)=\frac{1}{2 \pi}\left(4 p^{2} \rho^{-1}-\mu^{2}-\frac{5}{6} K\right)=\frac{1}{2 \pi}\left(\frac{10}{3} \frac{1}{\rho} \partial \bar{\partial} \phi-\mu^{2}+H^{2}\right)
$$

and the jacobian, 
I will derive the boson-fermion correspondence. From (3-18), the Ward-Takahashi identity $[29,30]$,

$$
\left.\frac{\delta}{\delta \alpha(q)}\left(Z_{1}-Z_{2}\right)\right|_{\alpha(q)=0} \equiv 0
$$

gives an anomaly,

$$
\rho^{-1} \delta_{a}^{\alpha} \partial_{\alpha}<\mathrm{i} \overline{\boldsymbol{\psi}} \sigma^{a} \rho^{1 / 2} \boldsymbol{\psi}>+<\overline{\boldsymbol{\psi}} \mathbf{D} \boldsymbol{\psi}>=\frac{1}{2 \pi} \mu^{2}+\frac{5}{12 \pi} K-\frac{1}{2 \pi}\left(H^{2}\right),
$$

where $\langle\mathcal{O}\rangle$ means the expectation value of $\mathcal{O}$ related to the partition function (3-1). I will refer this anomaly "immersed anomaly".

\section{$\S 4$. Discussion}

The right hand side of (3-38) is closely related to the conformal anomaly in the string theory and the Liouville action. If $H$ vanishes, the arguments in the previous section can be parallelled to the calculation of the conformal anomaly [22]. The case $H=0$ is known as the minimal surface in the immersion geometry $[2,11,27]$. Thus the quantity $\mu^{2}$ introduced in (3-31) is identified with the coupling constant of the dilaton in the Liouville action [22]. This picture preserves in the region with the finite constant curvature $H$ and then the physical meaning of $\mu^{2}$ is clarified.

Furthermore it should be noted that if I employ the heat kernel regularization instead of the modified negative power regularization, $\mu^{2}$ appears as infinite value, $\mu^{2} \sim 1 / \tau$. Thus mathematically

$\mu^{2}$ is interpreted as a convergence parameter which makes the kernel finite and this picture consists with the motivation to make the integral in (3-28) well-defined.

Here I will investigate the meanings of the anomalous relation (3-28) as follows. I will integrate both sides in (3-39),

$$
\int_{\Sigma} \mathrm{d}^{2} q\left(\mathrm{i} \delta_{a}^{\alpha} \partial_{\alpha}<\overline{\boldsymbol{\psi}} \sigma^{a} \rho^{1 / 2} \boldsymbol{\psi}>+\rho<\mathrm{i} \overline{\boldsymbol{\psi}} \not \boldsymbol{\psi}>+\frac{1}{2 \pi} \rho\left(H^{2}\right)-\frac{1}{2 \pi} \mu^{2} \rho-\frac{5}{12 \pi} \rho K\right)=0 .
$$

The first term is locally expressed as total derivative $j:=<\mathrm{i} \delta_{a}^{\alpha} \overline{\boldsymbol{\psi}} \sigma^{a} \rho^{1 / 2} \boldsymbol{\psi}>\mathrm{d} q^{\alpha}$. Thus let the surface $\Sigma$ be divided as

$$
\Sigma=\Sigma_{+} \cup \Sigma_{-}, \quad S^{1} \approx \Sigma_{+} \cap \Sigma_{-}, \quad \Sigma_{+} \approx \Sigma_{-} \approx \mathbb{R}^{2},
$$

where $\approx$ means the homeomorphism and I will define $j_{ \pm}$as functions over $\Sigma_{ \pm}$. Then the integration of the first term becomes,

$$
\left.\int_{\Sigma} \mathrm{d} * j=\int_{\partial \Sigma_{+}}\right] * j_{+}+\int_{\partial \Sigma_{-}} * j_{-}=\int_{\partial \Sigma_{+}}\left(* j_{+}-* j_{-}\right)=B_{2} \nu
$$

where $\nu$ is an integer and $B_{2}$ is a constant parameter. Thus it can be regarded as the candidacy of the generator of the fundamental group of $\Sigma_{+} \cap \Sigma_{-}$while the Euler characteristic $\chi$ expresses the global topology of the surface. If the current is conserved, $\nu$ vanishes.

Furthermore the third term means the area of the surface $\mathcal{S}$,

$$
A:=\int_{\Sigma} \rho \mathrm{d}^{2} q
$$

Using these quantities, I obtain the global expression of (3-38), 
Even though the current is not conserved, $B_{2} \nu$ is expected as a topological quantity. Thus (4-5) means that the expectation value of the action of the Dirac operator is written as the Willmore functional and the area of the surface. If the mean curvature vanishes, the minimal of area of the surface corresponds to stationary point of the action of the Dirac operator. This correspondence is theorem of the minimal surface and of old Weierstrass-Enneper equation [11]. For general immersion, investigation on the Dirac operator of the KKWE equation (3-9) means studying the Willmore surface itself if fixing the area. On the case of Schrödinger particle, the immersion effect appears as attractive potential and thus the sign of the Willmore action can be naturally interpreted [43]. Furthermore, since the Liouville action can be extended to that with supersymmetry [22], I believe that this correspondence (4-5) between these actions should be interpreted by supersymmetry of this system.

The Willmore surface problem of $\mathbb{R}^{3}$ has very similar structure of the elastica problem of $\mathbb{R}^{2}$. Corresponding to the Willmore functional (2-20), there is Euler-Bernoulli functional for an elastica $[10,21]$,

$$
E=\int \mathrm{d} q^{1} k^{2}
$$

where $k$ is a curvature of the elastica $[10,19]$. While the Willmore surface is related to the modified Novikov-Veselov (MNV) equation, the elastica is related to the modified KdV equation. From the soliton theory, the MNV equation is a higher dimensional analogue of the MKdV equation [6]. The Dirac operator appearing in the auxiliary linear problems of the MKdV equation is realized as the operator for the Dirac field confined in the elastica [28-32] as the KKWE equation might be related to auxiliary linear problems of the MNV equation $[2,6]$ and is realized as the equation of the Dirac field confined in the immersed surface [1].

In the series of works [28-32], I have been studying the elastica in terms of the quantized Dirac fields in the elastica. In terms of the partition function of the Dirac field, I constructed the JimboMiwa theory of the MKdV hierarchy [32] and showed the physical meaning of the inverse scattering method and the monodromy preserving deformation [28,31]. Investigation on the Dirac operator of the KKWE equation might lead us to the Sato-type theory of the MNV hierarchy.

Furthermore, recently I exactly quantized the elastica of the Euler-Bernoulli functional (4-6) preserving its local length and found that its moduli is closely related to the two-dimensional quantum gravity; the quantized elastica obeys the MKdV hierarchy and at a critical point, the Painlevé equation of the first kind appears [21]. Instead of the local length preserving, after imposing that the surface preserves its complex structure or other constraints e.g. $\rho H=$ constant [24,25], one could quantize the Willmore functional and then, I expect that the MNV hierarchy might appear [44] as the quantized motion of a Willmore surface in the path integral as the MKdV hierarchy appears in the quantization of the elastica [21].

Moreover recently another relation between the geometry and quantum equation, was discovered by Konopelchenko [45-47]. At this stage, I could not physically interpret the new relation but believe that there is another quantum meanings. I expect that his old and new relations $[2-4,44-46]$ are clarified in the quantum mechanical context.

\section{Acknowledgment}

I would like to thank Prof. S. Saito, for critical discussions and continuous encouragement. I am grateful to Prof. Y. Ohnita for telling me the ref.[5] and ref.[44] and to Prof. B. G. Konopelchenko for sending me his very interesting works and encouragement. I would also like to thank Prof. T. Tokihiro, Prof. K. Sogo, Y. Onishi and Prof. K. Tamano for helpful discussions at the earliest stage and continuous encouragement. 
3. B. G. Konopelchenko and I. A. Taimanov, J. Phys. A: Math. \& Gen. 29 (1996), 1261-65.

4. B. G. Konopelchenko and I. A. Taimanov, Generalized Weierstarass formulae, soliton equations and Willmore surfaces I. Tori of revolution and the mKDV equation, dg-ga/9506011.

5. K. Kenmotsu, Math. Ann. 245, 89-99.

6. I. A. Taimanov, Modified Novikov-Veselov equation and differential geometry of surface, dg-ga/9511005.

7. I. A. Taimanov, Surface revolution in terms of soliton, dg-ga/9610013.

8. N. Berline, E. Getzler and M. Vergne,, Heat Kernels and Dirac Operators, Springer, Berlin, 1991.

9. G. Faltings, Lectures on the Arithemtic Riemann-Roch theorem, Princeton Univ. Press, Princeton, 1992.

10. C. Truesdell, Bull. Amer. Math. Soc. 9 (1983), 293-310.

11. A. I. Bobenko, Surfaces in terms of 2 by 2 matrices: Old and new integrable cases, Harmonic Maps and Integrable Systems (A. P. Fordy and J. C. Wood, eds.), Vieweg, Wolfgang Nieger, 1994.

12. R. E. Goldstein and D. M. Petrich, Phys. Rev. Lett. 67 (1991,), 3203-3206.

13. R. E. Goldstein and D. M. Petrich, Phys. Rev. Lett. 67 (1992,), 555-558.

14. S. Matsutani, Int. J. Mod. Phys. A 10 (1995), 3109-3130.

15. A. Doliwa, P. M. Santini, Phys. Lett. A 185 (1994), 373-384.

16. A. Bobenko and U. Pinkall,, J. Diff. Geom. 43 (1996), 527-611.

17. H. C. Wente, Pacific J. Math 121 (1986), 193-243.

18. U. Abresh, J. reine u. angew Math. 374 (1987), 169-192.

19. U. Pinkall and I. Sterling, Ann. Math 130 (1989), 407-451.

20. A. P. Fordy and J. C. Wood, Harmonic Maps and Integrable Systems, Vieweg, Wolfgang Nieger, 1994.

21. S. Matsutani, solv-int/9707003.

22. A. M. Polyakov, Gauge Fields and Strings, Harwood Academic Publishers, London, 1987.

23. J-L. Gervais and Y. Matsuo, Com. Math. Phys. 152 (1993), 317-368.

24. R. Carroll and B. Konopelchenko, Int. J. Mod. Phys. A11 (1996), 1183-1216.

25. K. S. Viswanathan and R. Parthasarathy, Ann. Phys. 244, 241-261.

26. T. J. Willmore, J. Lond. Math. Soc. 2 (1971), 307-310.

27. T. J. Willmore, Riemannian Geometry, Oxford, Oxford, 1993.

28. S. Matsutani and H. Tsuru, Phys. Rev A 46 (1992), 1144-7.

29. S. Matsutani, Prog. Theor. Phys. 91 (1994), 1005-37.

30. S. Matsutani, J. Phys. A: Math. \& Gen. 28 (1995), 1399-1412.

31. S. Matsutani, Thesis in Tokyo Metropolitan Univ., 1995.

32. S. Matsutani, Int. J. Mod. Phys. A 10 (1995), 3109-3130.

33. K. Fujikawa, Phys. Rev. Lett. 42, 1195-1199.

34. A. P. Balachandran, G. Marmo, V. P. Nair and C. G. Trahern, Phys Rev D 25, 2713-2718.

35. M. S. Alves, C. Farina and C. Wotzasek, Phys. Rev. D 43, 4145-4147.

36. E. Elizalde, S. D. Odintsov, A. Romeo, A. A. Bytsenko and S. Zerbini, Zeta Regularization Techniques with Apprications, World Scientific, Singapore, 1994.

37. M. F. Atiyah and I. M. Singer, Ann. of Math. 87 (1968), 484-530.

38. M. F. Atiyah and I. M. Singer, Ann. of Math. 87 (1968), 546-604.

39. P. B. Gilkey, Invariance Theory, The Heat Equation and the Atiyah-Singer Index Theorem, Publish or Perish, Wilmington, 1984.

40. A. E. H. Love, A Treatise on the Mathematical Theory of Elasticity, Cambridge Univ. Press, Cambridge, 1927.

41. P. Ramond, Field Theory: A Modern Primer, Benjamin, Mento Park, 1981.

42. M. Burgess and B. Jensen, Phys. Rev. A 48 (1993), 1861-6.

43. S. Matsutani, J. Phys. A: Math. \& Gen. 26 (1993), 5133-5143.

44. P. G. Grinevich and M. U. Schmidt, Conformal invariant functionals of immersioons of tori into $\mathbb{R}^{3}$, dgga/9702015.

45. B. G. Konopelchenko, Inverse Problem 12 (1996), L13-L18.

46. B. G. Konopelchenko, J. Math. Phys 38 (1997), 434-543.

47. R. Beutler and B. G. Konopelchenko, Surfaces of Revolution via the Schrödinger Equation: Construction, Integrable Dynamics and Visualization (1996). 